\title{
Perfil do risco cardiovascular de pacientes diabéticos atendidos em ambulatório de especialidades
}

\author{
Profile of cardiovascular risk in diabetic patients in a center of specialties
}

\author{
Daniele Botelho Vinholes ${ }^{1}$, Heloísa Alves Pacheco ${ }^{2}$
}

\section{RESUMO}

Objetivo: Identificar o perfil de risco cardiovascular em pacientes diabéticos atendidos no Ambulatório Médico de Especialidades em Tubarão.

Materiais e Métodos: Realizou-se um estudo retrospectivo composto por 101 pacientes diabéticos. A coleta foi realizada a partir de dados contidos nos prontuários médicos dos pacientes. Foram consideradas para o estudo as seguintes variáveis: sexo, idade, tipo de diabetes, tempo de diagnóstico da doença, tabagismo, índice de massa corporal (IMC), glicemia, hemoglobina glicada (HbA1c), perfil lipídico e microalbuminúria.

Resultados: A maioria da amostra foi composta por mulheres, a média de idade foi de 59,6 anos (dp $\pm 12,5)$, o subtipo mais comum foi o tipo 2 e a média de diagnóstico da doença foi de 14,1 anos (dp $\pm 10,3$ ). Dos participantes, $11,9 \%$ relataram serem fumantes e $56,4 \%$ apresentavam-se obesos. A hemoglobina glicada estava alterada em $74,3 \%$ dos indivíduos. Das características que compõem o perfil lipídico, mais da metade apresentou esses parâmetros alterados com exceção do colesterol total que estava controlado em $63,4 \%$ dos diabéticos. A microalbuminúria estava controlada em $58,4 \%$ dos pacientes.

Conclusão: A maioria dos pacientes diabéticos atendidos neste serviço de saúde apresentavam parâmetros alterados, relacionando-se com aumento do risco de desenvolver doenças cardiovasculares.

Palavras-chave: diabetes mellitus; doenças cardiovasculares; prevenção secundária.

\section{ABSTRACT}

Objective: To identify the cardiovascular risk profile in diabetic patients attended in the Ambulatory of Medical Specialties in Tubarão.

Materials and Methods: Retrospective study with 101 diabetic patients. Data collection was performed by using the medical records of patients. The following variables were used: gender, age, type of diabetes, smoking, time since disease diagnosis, body mass index, glycemia, glycated hemoglobin, lipid profile and microalbuminuria.

Results: The majority of individuals were women with an average age of 59.6 years (sd \pm 12.5 ). The most common diabetes subtype was type 2 and the average disease diagnosis time was 14.1 years (sd \pm 10.3 ). $11.9 \%$ of the patients were smokers and $56.4 \%$ were obese. The glycated hemoglobin was abnormal in $74.3 \%$ of them. Considering the characteristics of the lipid profile, the majority had alterations on lipid parameters, except for the total cholesterol that was controlled in $63.4 \%$ of diabetic patients. Microalbuminuria was normal in $58.4 \%$ of patients.

Conclusions: It was demonstrated that the majority of diabetic patients attended in this ambulatory presented alterations in parameters that were associated with increased risk of developing cardiovascular disease.

Keywords: diabetes mellitus; cardiovascular diseases; secondary prevention.

\footnotetext{
${ }^{1}$ Nutricionista. Doutora em Epidemiologia pela UFRGS. Professora do curso de Medicina da Universidade do Sul de Santa Catarina (UNISUL).

${ }^{2}$ Acadêmica do Curso de Medicina da UNISUL.
} 


\section{INTRODUÇÃO}

O Diabetes Mellitus (DM) é uma doença crônica caracterizada por níveis anormais de glicose plasmática $^{1,2}$ resultante da secreção inadequada de insulina, da inabilidade de sua ação ou de ambas as características $^{2}$.

Diversos mecanismos estão relacionados ao desenvolvimento do DM. No diabetes tipo 1, há a destruição das células $\beta$ pancreáticas por ação imunológica levando a redução total da secreção de insulina. Dentro deste subtipo da doença está incluída a Latent Autoimmune Diabetes of the Adults (LADA), cuja destruição das células $\beta$ pancreáticas ocorre de maneira mais lenta definindo sintomas mais tardiamente ${ }^{3}$. Já o diabetes tipo 2 que representa a forma mais prevalente, caracteriza-se por uma diminuição gradativa da secreção de insulina associada à resistência da sua ação nos tecidos corporais ${ }^{2}$. Além dos subtipos clássicos da doença, há também os tipos específicos de diabetes identificados por defeitos genéticos nas células do pâncreas produtoras de insulina, como o MaturityOnset Diabetes of the Young (MODY) em que a ação insulínica nos tecidos não está alterada, entretanto, há uma deficiência na secreção de insulina ${ }^{3}$. O diabetes pode ser causado também por doenças genéticas específicas, endocrinopatias, doenças exócrinas do pâncreas ou ser desencadeada durante a gestação ${ }^{3}$.

De acordo com dados da World Health Organization (WHO), a estimativa para 2030 é que a prevalência mundial de diabetes seja superior a $4,4 \%$ entre indivíduos de diferentes grupos etários ${ }^{4}$, no entanto existe uma tendência global de aumento desta prevalência podendo se tornar uma pandemia nos próximos anos. Este crescente aumento pode estar relacionado ao envelhecimento da população e ao aumento da incidência de obesidade e sedentarismo ${ }^{5}$. No Brasil, de acordo com uma pesquisa realizada em uma capital, a prevalência de diabéticos foi de $4,9 \%$ entre indivíduos adultos ${ }^{6}$ diferenciando-se de estudos em outras cidades cuja prevalência de DM oscilava em torno de $13,5 \%$.

A manutenção do nível glicêmico elevado cronicamente provoca dano, disfunção e falência de diversos sistemas corpóreos ${ }^{2}$. A evolução da doença associada a uma adesão ineficaz ao tratamento pode comprometer os vasos de grande calibre provocando doenças macrovasculares e os vasos de pequeno calibre desencadeando doenças microvasculares principalmente na retina, nos rins e no sistema nervoso periférico ${ }^{1}$. Em um estudo desenvolvido na região sul do país, verificou-se que a complicação macrovascular mais frequente em pacientes com diabetes tipo 2 foi a cardiopatia isquêmica presente em $36 \%$ dos diabéticos, também foi encontrado na pesquisa uma porcentagem significativa da amostra com acometimento retiniano, renal e nervoso ${ }^{9}$.

Em virtude do acometimento sistêmico do diabetes, é sabido que essa doença aumenta o risco para desenvolvimento de doença cardiovascular assemelhando-se ao aumento de risco ocasionado após infarto do miocárdio ${ }^{10}$. O risco cardiovascular em uma população diabética pode chegar a $18,2 \%$ e esse elevado risco de desenvolvimento de doenças cardiovasculares ${ }^{11}$ ocorre em virtude da aceleração do processo de formação de placas ateroscleróticas por acúmulo de partículas de lipoproteína de baixa densidade (LDL) na parede dos vasos sanguíneos que desenvolvem cronicamente lesão endotelial e inflamação no local ${ }^{2}$.

Devido o aumento do risco cardiovascular em pacientes diabéticos é importante que haja um tratamento médico contínuo associado à educação do paciente acerca da própria doença, pois a partir desses componentes consegue-se reduzir ou evitar as complicações durante o curso da doença ${ }^{2}$.

Visto que determinados fatores provocam aumento do risco para doenças cardiovasculares, é importante a identificação desses componentes para auxiliar no melhor manejo desses pacientes, garantindo um aumento da sobrevida e, também, um aumento da qualidade de vida. O diabetes é um fator de risco cardiovascular independente, aumentando em 2 a 4 vezes o risco de desenvolvimento de doença cardiovascular $^{12}$. Consequentemente se houver um controle adequado da doença associado a uma intervenção sobre os outros fatores de risco cardíacos e vasculares poder-se-ia contribuir para uma melhoria na qualidade de vida desses indivíduos reduzindo o risco 
de complicações cardíacas e vasculares que aumentam a morbidade e a mortalidade desses pacientes.

Com base no exposto acima, este estudo objetiva identificar o perfil de risco cardiovascular em pacientes diabéticos atendidos em Ambulatório Médico de Especialidades do município de Tubarão-SC; assim como identificar o perfil sociodemográfico desses pacientes, os subtipos de diabetes mais frequentes, a presença de tabagismo e de excesso de peso e a alteração de exames laboratoriais que influenciam no aumento da chance de desenvolver doença cardiovascular. Além disso, verificar a possível associação entre as variáveis estudadas.

\section{MATERIAIS E MÉTODOS}

O presente estudo caracteriza-se por um delineamento retrospectivo. A população em estudo incluiu todos os prontuários de pacientes com diagnóstico de DM atendidos no Ambulatório Médico de Especialidades (AME) de Tubarão-SC no período entre janeiro de 2010 a maio de 2011. Foram excluídos os pacientes com dados laboratoriais com data superior a um ano e o preenchimento inadequado do prontuário. Inicialmente foram selecionados 137 prontuários para o estudo, entretanto essa amostra reduziu-se a 101 pacientes após observados os critérios de exclusão do estudo, totalizando $73,72 \%$ do total inicial.

A coleta dos dados foi feita a partir de informações contidas nos prontuários de pacientes diabéticos atendidos no Ambulatório Médico de Especialidades e para o desenvolvimento do estudo foi elaborado um instrumento contendo as principais características de interesse para facilitar a coleta de dados do estudo. Foram consideradas variáveis do estudo: idade, sexo, tipo de diabetes, tempo de diagnóstico da doença, tabagismo, índice de massa corporal (IMC), glicemia de jejum, hemoglobina glicada $(\mathrm{HbA} 1 \mathrm{c})$, colesterol total, triglicerídeos, colesterol LDL, colesterol HDL e microalbuminúria. Para o IMC adotou-se a unidade de medida $\mathrm{kg} / \mathrm{m}^{2}$ e posteriormente foi classificado em: eutrófico (IMC: $18,5-24,9 \mathrm{~kg} / \mathrm{m}^{2}$ ), sobrepeso (IMC: 25 $29,9 \mathrm{~kg} / \mathrm{m}^{2}$ ) e obeso (IMC acima de $30 \mathrm{~kg} / \mathrm{m}^{2}$ ), de acordo com a Organização Mundial da Saúde

Em relação aos parâmetros laboratoriais, para glicemia de jejum, colesterol total, colesterol LDL, colesterol HDL e triglicerídeos considerou-se a unidade de medida $\mathrm{mg} / \mathrm{dL}$; a variável hemoglobina glicada (HbA1c) foi descrita em percentual e a microalbuminúria em mg/24 horas. Para a variável HbA1c foi considerado controle valores iguais ou inferiores a $7 \%$; o colesterol total foi considerado controlado quando o valor era igual ou inferior a $200 \mathrm{mg} / \mathrm{dL}$ e os triglicerídeos estavam dentro dos parâmetros de controle quando em valores iguais ou inferiores a $150 \mathrm{mg} / \mathrm{dL}$. O colesterol LDL foi considerado controlado quando os valores foram igual ou abaixo de $100 \mathrm{mg} / \mathrm{dL}$ e o colesterol HDL estava controlado em homens quando acima de $40 \mathrm{mg} / \mathrm{dL}$ e controlado em mulheres quando acima de $50 \mathrm{mg} / \mathrm{dL}$. Em relação à microalbuminúria em 24 horas, eram considerados os valores normais abaixo de $30 \mathrm{mg} / 24$ horas, a microalbuminúria era entre $30-300 \mathrm{mg} / 24$ horas e macroalbuminuria era acima de $300 \mathrm{mg} / 24$ horas.

O projeto foi submetido à avaliação do Comitê de Ética em Pesquisa (CEP) da UNISUL com o protocolo de aprovação 12.025.4.01-III e segue as diretrizes e normas regulamentadoras de pesquisa envolvendo seres humanos propostas pela Resolução do Conselho Nacional de Saúde no 196/1996.

Para a análise dos dados foi elaborado um banco de dados no programa Epinfo 3.5.1, sendo que para análise estatística foi utilizado o programa SPSS versão 20,0. Foi realizada análise descritiva utilizando frequência e percentual para as variáveis categóricas e a média ou mediana e desvio padrão para as variáveis numéricas. Para a análise bivariada foram utilizados os seguintes testes estatísticos: qui-quadrado para as variáveis categóricas e teste $\mathrm{t}$ de Student para a comparação de médias. O nível de significância utilizado foi de $5 \%$.

\section{RESULTADOS}

Foram analisados os prontuários de 101 pacientes com diagnóstico de DM, dos quais $65,3 \%$ pertenciam ao sexo feminino, com média de idade de 59,6 anos $(\mathrm{DP} \pm 12,5)$. Em relação ao tipo de diabetes, $94,1 \%$ de diabetes tipo 2 e o tempo de diagnóstico da doença teve média de 14,1 anos $(\mathrm{DP} \pm 10,3)$ e a mediana 11 anos, sendo o tempo mínimo de diagnóstico de 1 ano e 
TABELA 1 - Características sociodemográficas e perfil de risco cardiovascular de pacientes atendidos em um Ambulatório no Sul de Santa Catarina.

\begin{tabular}{|c|c|}
\hline Características & $\begin{array}{l}\text { n (\%) ou Média } \\
\text { (Desvio padrão) }\end{array}$ \\
\hline \multicolumn{2}{|l|}{ Sexo } \\
\hline Masculino & $35(34,7)$ \\
\hline Feminino & $66(65,3)$ \\
\hline \multicolumn{2}{|l|}{ Idade (em anos) } \\
\hline Até 30 & $3(3,0)$ \\
\hline $30-40$ & $4(4,0)$ \\
\hline $40-50$ & $13(12,9)$ \\
\hline Acima de 50 & $81(80,2)$ \\
\hline \multicolumn{2}{|l|}{ Tipo de diabetes } \\
\hline Tipo 1 & $4(4)$ \\
\hline Tipo 2 & $94(94,1)$ \\
\hline LADA & $1(1)$ \\
\hline MODY & $1(1)$ \\
\hline Tempo de diagnóstico (em anos) & $14,10(10,33)$ \\
\hline Tabagismo & $12(11,9)$ \\
\hline \multicolumn{2}{|l|}{ Índice de massa corporal } \\
\hline Eutrófico & $18(17,8)$ \\
\hline Sobrepeso & $26(25,7)$ \\
\hline Obesidade & $57(56,4)$ \\
\hline Glicemia de jejum (mg/dL) & $145(77,03)$ \\
\hline Hemoglobina glicada (\%) & $8,42(2,27)$ \\
\hline \multicolumn{2}{|l|}{ Perfil lipídico } \\
\hline Colesterol total (mg/dL) & $185,8(51,4)$ \\
\hline Colesterol LDL (mg/dL) & $105,88(41,92)$ \\
\hline Triglicerídeos(mg/dL) & $175,4(99,57)$ \\
\hline \multicolumn{2}{|c|}{$\begin{array}{l}\text { o máximo de } 46 \text { anos. } \\
\text { Quanto ao tabagismo, em } 11,9 \% \text { havia registro de ser } \\
\left.\text { fumante, a média de IMC foi de } 30,7 \mathrm{~kg} / \mathrm{m}^{2} \text { (DP } \pm 6,0\right) \text {, } \\
\text { sendo o mínimo valor encontrado de } 20,1 \mathrm{~kg} / \mathrm{m}^{2} \text { e o } \\
\text { máximo de } 50 \mathrm{~kg} / \mathrm{m}^{2} \text {. Entre o total de pacientes do } \\
\text { estudo, } 56,4 \% \text { apresentavam-se obesos, sem } \\
\text { diferenciação do grau de obesidade. De acordo com a } \\
\text { glicemia de jejum, a mediana foi de } 145 \mathrm{mg} / \mathrm{dL} \text { sendo o } \\
\text { mínimo de glicemia encontrada de } 70 \mathrm{mg} / \mathrm{dL} \text { e o máximo } \\
\text { de } 460 \mathrm{mg} / \mathrm{dL} \text {. Em relação à hemoglobina glicada, a } \\
\text { média foi de } 8,4 \% \text { (DP } \pm 2,3 \text { ). As demais características } \\
\text { estão descritas na tabela } 1 \text {. }\end{array}$} \\
\hline
\end{tabular}

Aproximadamente $50 \%(n=50)$ dos pacientes estavam com os índices de Triglicerídeos e Colesterol-LDL controlados. Para o colesterol total, o percentual de controle atingido foi de $63,4 \%$ e para o Colesterol-HDL foi de $35,6 \%$.

Nas tabelas 2 e 3 são apresentados os resultados dos testes da análise bivariada. Não houve associação estatisticamente significativa entre o controle da diabete através da hemoglobina glicada e as variáveis sexo, idade e IMC

\section{DISCUSSÃO}

Neste estudo encontrou-se maior prevalência do gênero feminino com diagnóstico de DM assemelhandose a estudos realizados em diferentes municípios do Brasil $^{8,13}$, entretanto em estudo realizado em Ribeirão Preto, apesar de as mulheres serem em maior número, a prevalência foi consideravelmente maior que na presente pesquisa correspondendo a $71,3 \%$ da amostra $^{14}$. O fato de as mulheres serem mais prevalentes no estudo pode ser explicado em virtude da maior procura desse gênero ao serviço de saúde. No entanto, em estudo realizado em Minas Gerais foi encontrado um risco maior de mulheres desenvolverem diabetes em relação aos homens ${ }^{15}$ provavelmente em virtude das alterações metabólicas que ocorrem durante o climatério e a menopausa que predispõem a chance de desenvolver DM.

A média de idade entre os participantes do estudo foi muito similar a um estudo realizado na região nordeste ${ }^{8}$, sendo que a alta média de idade pode ser em decorrência da maior prevalência de diabetes tipo 2 cuja evolução se dá mais tardiamente ${ }^{3}$.

Como em pesquisa realizada no município de Cuiabá $^{13}$, o presente estudo evidenciou maior prevalência de diabetes tipo 2 na população estudada. Essa característica corrobora com a tendência mundial ${ }^{2}$ de alta prevalência de diabetes tipo 2, visto o aumento da expectativa de vida, da obesidade e do sedentarismo. No presente estudo houve uma prevalência de $1 \%$ de pacientes com subtipo LADA. Em uma revisão sobre 0 assunto foi encontrado uma prevalência desse subtipo entre indivíduos com diabetes 
TABELA 2 - Análise bivariada entre o controle da diabetes e idade e índice de massa corporal de pacientes atendidos em ambulatório de especialidades (teste t de Student).

\begin{tabular}{lcccccc}
\hline Hemoglobina glicada & \multicolumn{3}{c}{ IMC } & \multicolumn{3}{c}{ Idade } \\
\hline & Média & Desvio padrão & Valor $p$ & Média & Desvio padrão & Valor $p$ \\
Controlado & 30,62 & 4,92 & 0,91 & 57,08 & 13,57 & 0,23 \\
Não controlado & 30,77 & 6,41 & & 60,45 & 12,11 & \\
\hline
\end{tabular}

tipo 2 de 10\%16. O diabetes tipo MODY apresentou 1 caso no presente estudo, diferente de estudo realizado no Reino Unido que de 112 crianças estudados 20 apresentavam diabetes tipo MODY ${ }^{17}$.

Em relação ao tempo de diagnóstico da doença, no presente estudo foi encontrado um tempo médio de diagnóstico de 14,1 anos (DP \pm 10,3) diferentemente do estudo de Ribeirão Preto que obteve um tempo médio de diagnóstico de 8,7 anos $(\mathrm{DP} \pm 6,7)^{14}$. Pode-se explicar essa diferença, pois o estudo conduzido em Ribeirão Preto considerou apenas os pacientes com diabetes cujo diagnóstico normalmente ocorre tardiamente devido a ausência de manifestações clinicas na fase inicial da doença e consequentemente o rastreio da doença não ocorre de maneira eficaz. No presente estudo foram avaliados todos os pacientes, inclusive os pacientes que apresentam diabetes tipo 1 e tipos mais específicos de diabetes, o que aumentaria o tempo de diagnóstico da doença visto que o diabetes tipo 1 normalmente tem como primeira manifestação um quadro de cetoacidose diabética em decorrência da total destruição das células $\beta$ pancreáticas ${ }^{3}$.

Em relação ao hábito de fumar o presente estudo apresentou índice menor em relação à prevalência de fumantes no Brasil e na região Sul, respectivamente de $15,1 \%$ e $17,9 \%^{18}$, entretanto esta pesquisa faz um levantamento de dados de toda população brasileira diferente do nosso estudo que considera uma amostra isolada da população. Outro estudo realizado no Rio Grande do Sul apresentou uma prevalência consideravelmente superior de indivíduos fumantes diabéticos chegando a $30,9 \%{ }^{19}$.

De acordo com a classificação do IMC, o total de obesos no presente estudo foi relativamente maior que em estudo realizado no estado do Rio Grande do Sul cuja prevalência de obesidade entre diabéticos foi de $35,3 \%{ }^{19}$.
A média de glicemia encontrada no nosso estudo foi menor que em outro estudo que foi de $165 \mathrm{mg} / \mathrm{dL}$ $(\mathrm{DP} \pm 72)^{11}$. No entanto, a glicemia de jejum não constitui um parâmetro fidedigno para avaliar o controle da doença e, portanto, avaliar o risco futuro de complicações $^{2}$. A hemoglobina glicada é considerada um parâmetro mais confiável para avaliar o controle da doença, pois não sofre alteração de acordo com as modificações na dieta do paciente ${ }^{2}$. A média de hemoglobina glicada foi similar a obtida na avaliação inicial sem intervenção de estudo elaborado com diabéticos tipo 2 em Belo Horizonte ${ }^{20}$. No nosso estudo houve uma grande parcela da amostra que não apresentou hemoglobina glicada dentro dos parâmetros desejáveis para doença implicando em aumento do risco de desenvolver doenças cardiovasculares futuramente.

No estudo de Silva et al. ${ }^{21}$, os parâmetros que compõem o perfil lipídico apresentaram-se alterados na maioria dos indivíduos, fato similar ocorreu no nosso estudo exceto pela variável colesterol total que esteve dentro dos limites de normalidade na maioria dos pacientes. Pode-se explicar esta diferença visto que a amostra que compunha aquele estudo era de pacientes idosos apresentando apenas diabetes tipo 2, normalmente idosos apresentam perfil lipídico alterado em relação ao resto da população bem como indivíduos com diabetes tipo $2^{21}$.

Segundo o estudo de Scheffel et al. ${ }^{9}$, a prevalência de doença renal crônica entre indivíduos com diabetes tipo 2 foi de $37 \%$ muito semelhante a prevalência encontrada no nosso estudo. O parâmetro utilizado para avaliar presença de doença renal foi a microalbuminúria que constitui isoladamente fator de risco para desenvolver doença cardiovascular ${ }^{22}$.

O presente estudo teve por objetivo avaliar o risco cardiovascular de pacientes diabéticos a partir de 
TABELA 3 - Análise bivariada entre o controle da diabetes e sexo de pacientes atendidos em ambulatório de especialidades ( $n(\%)$ - teste qui-quadrado).

\begin{tabular}{lccc}
\hline Sexo & \multicolumn{2}{c}{ Hemoglobina glicada } & Valor $\mathbf{p}$ \\
\hline & Controlado & Não controlado & 0,15 \\
Masculino & $6(17,1)$ & $29(82,9)$ & \\
Feminino & $20(30,3)$ & $46(69,7)$ & \\
\hline
\end{tabular}

informações contidas em prontuários médicos. É importante ressaltar que o presente estudo apresenta algumas limitações, visto que os dados foram obtidos a partir de informações previamente coletadas, não sendo possível um controle maior dessas informações por parte dos pesquisadores. Além disso, o estudo foi elaborado em um grupo específico de uma população com uma amostra limitada, portanto as características do estudo não necessariamente irão refletir as características de uma grande população.

Portanto, este estudo pode demonstrar que grande parte dos pacientes diabéticos atendidos neste serviço de saúde era do gênero feminino concordando com a maioria dos estudos, pertenciam ao subtipo 2 que é o mais prevalente mundialmente de acordo com estudos e consensos, eram obesos sinalando a tendência global de aumento da obesidade, eram tabagistas uma pequena parcela do estudo comparando-se com a prevalência brasileira e apresentam fatores de risco que aumentavam a chance de desenvolver doença cardiovascular, visto que os valores são bastante significativos na maioria dos parâmetros utilizados.

\section{REFERÊNCIAS}

1. Fowler MJ. Microvascular and macrovascular complications of diabetes. Clin Diabetes 2011; 29(3): 11622.

2. American Diabetes Association. Standards of Medical Care in Diabetes - 2012. Diabetes Care 2012; 35(1): 11 . 63.

3. American Diabetes Association. Diagnosis and classification of diabetes mellitus. Diabetes Care 2012; 35 S1: S64-S71.

4. Wild SH, Roglic G, Green A, Sicree R, King H. Global Prevalence of Diabetes: Estimates for the Year 2000 and Projections for 2030. Diabetes Care 2004; 27(10): 2569.

5. Rato Q. Diabetes Mellitus: um problema de saúde global Rev Port Cardiol 2010; 29 (4): 539-43.

6. Mielczarski RG, Costa JSD, Olinto MTA. Epidemiologia e organização de serviços de saúde: diabetes mellitus numa comunidade de Porto Alegre. Ciênc Saúde Colet 2012; 17(1): 71-8.

7. Bosi PL, Carvalho AM, Contrera D, Casale G, Pereira MA, Gronner MF, Diogo TM, Torquarto MTCG, Oishi J, Leal
AMO. Prevalência de diabetes melito e tolerância à glicose diminuída na população urbana de 30 a 79 anos da cidade de São Carlos, São Paulo. Arq Bras Endocrinol Metab 2009; 53(6): 726-32.

8. Lyra R, Silva RS, Junior RMM, Matos MVC, Cézar NJB, Silva LM. Prevalência de diabetes melito e fatores associados em população urbana adulta de baixa escolaridade e renda do sertão nordestino brasileiro. Arq Bras Endocrinol Metab 2010; 54(6): 560-6.

9. Scheffel RS, Bortolanza D, Weber CS, Costa LA, Canan LH, Santos KG, Crispim D, Roisenberg I, Lisbôa HRK, Tres GS, Tschiedel B, Gross JL. Prevalência de complicações micro e macrovasculares e de seus fatores de risco em pacientes com diabetes melito do tipo $2 \mathrm{em}$ atendimento ambulatorial. Rev Med Bras 2004; 50(3): 2637.

10. Juutilainen A, Lehto $S$, Onnemaa $T$, Al'A K, Laakso M. Type 2 Diabetes as a "Coronary Heart Disease Equivalent". Diabetes Care 2005; 28(12): 2901-7.

11. Oliveira DS, Tannus LRM, Matheus ASM, Corrêa FH, Cobas RC, Cunha EF, Gomes MB. Avaliação do risco cardiovascular segundo os critérios de Framingham em pacientes com diabetes tipo 2. Arq bras endocrinol metab 2007; 51(2): 268-74.

12. Preis SR, Hwang SJ, Coady S, Pencina MJ, D’Agostino RB, Savage PJ, Levy D, Fox CS. Trends in all-cause and cardiovascular disease mortality among women and men with and without diabetes mellitus in the Framingham Heart Study, 1959 to 2005. Circulation. 2009; 119(13):1728-35.

13. Ferreira CLRA, Ferreira MG. Características epidemiológicas de pacientes diabéticos da rede pública de saúde - análise a partir do sistema HiperDia. Arq Bras Endocrinol Metab 2009; 53(1): 80-6.

14. Vigo KO, Torquato MTCG, Silvério IAS, Queiroz FA, Guanilo MCLTU, Pace AE. Caracterização de pessoas com diabetes em unidades de atenção primária $e$ secundária em relação a fatores desencadeantes do pé diabético. Acta Paul enferm 2005; 19(3): 296-303

15. Fidelis LC, Moreira OC, Teodoro BG, Oliveira CEP Prevalência de diabetes mellitus no município de Teixeiras-MG. Rev Bras Ativ Fís Saúde 2009; 14(1): 23-7.

16. Stenstrom G, Gottsater A, Bakhtadze E, Berger B, Sundkvist G. Latent Autoimmune Diabetes in Adults definition, prevalence, $\beta$-cell function, and treatment. Diabetes 2005; 54(2): S68-72.

17. Etisham S, Hattersley AT, Dunger DB, Barrett TG. First UK survey of pediatric type 2 diabetes and MODY. Arch Dis Child 2004; 89:526-9.

18. Barros AJD, Cascaes AM, Wehrmeister FC, Mesa JM, Menezes AMB. Tabagismo no Brasil: desigualdades regionais e prevalência segundo características ocupacionais. Ciênc Saúde Colet 2011; 16(9): 3707-16.

19. Schaan BDA, Harzheim E, Gus I. Perfil de risco cardíaco no diabetes mellitus e na glicemia de jejum alterada. Rev Saúde Publica 2004; 38(4): 529-36.

20. Torres HC, Franco LJ, Stradioto MA, Hortale VA, Schall VT. Avaliação estratégica de educação em grupo e individual no programa educativo em diabetes. Rev Saúde 
Pública 2009; 43(2): 291-8.

21. Silva RCP, Simões MJS, Leite AA. Fatores de risco para doenças cardiovasculares em idosos com diabetes mellitus tipo 2. Rev Ciên Farm Básica Apl 2007; 28(1): 113-21.

22. Klausen $\mathrm{K}$, Johnsen $\mathrm{KB}$, Rasmussen BF, Jensen $\mathrm{G}$, Clausen P, Scharling H, Appleyard M, Jensen JS. Very Low Levels of microalbuminuria are associated with increased risk of coronary heart disease and death independently of renal function, hypertension, and diabetes. Circulation 2004; 110:32-5.
Endereço para correspondência:

Daniele Botelho Vinholes

Rua Guararapes n 70, Apto. 701

Porto Alegre/RS - CEP 90690-340

Telefone: +55 5192627426

E-mail:dvinholes@terra.com.br 